\title{
Oestrogen plus progestin doubles the risk of dementia in post-menopausal women
}

\author{
Shumaker $S$, Legault $C$, Rapp $S$ et al. Estrogen plus progestin and the incidence of dementia and mild cognitive \\ impairment in postmenopausal women: the women's health initiative memory study: a randomized controlled trial. \\ JAMA 2003;289:2651-2661.

\section{QUESTION: Does oestrogen plus progestin increase the risk of dementia or mild cognitive impairment among post-menopausal women?}

\section{Design}

Randomised double blind placebo controlled trial.

\section{Setting}

39 Women's Health Initiative Clinical Centres, USA; May 1996 to December 1999.

\section{Participants}

4532 post-menopausal women aged at least 65 years and free of dementia at study entry.

\section{Intervention}

$0.625 \mathrm{mg}$ of conjugated equine oestrogen plus $2.5 \mathrm{mg}$ of medroxyprogesterone acetate (hormone therapy group), or placebo.

\section{Main outcome measures}

Dementia or mild cognitive impairment according to DSM-IV criteria. Clinical assessment of dementia or mild cognitive impairment was assessed by an approved clinician using a structured protocol.

\section{Main results}

Hormone therapy significantly increased the risk of dementia compared with placebo after a mean follow up of about 4 years, although the absolute risks were low in both groups $(40 / 2229$ [1.8\%] with hormone therapy $v$ 21/2303 [0.9\%] with placebo; ARI 0.89\%; hazard ratio $2.05 ; 95 \%$ CI 1.21 to 3.48 ). There was no significant difference in the rate of mild cognitive impairment between hormone therapy and placebo (hazard ratio $1.07 ; 95 \%$ CI 0.74 to 1.55$)$.

\section{Conclusions}

Hormone therapy comprising oestrogen and progestin increases the risk of dementia. Other studies have demonstrated that hormone therapy increases risks of heart disease, stroke, pulmonary embolism and breast cancer in post-menopausal women. Given these findings, the risks of hormone replacement therapy outweigh the benefits.

\section{Source of funding: \\ This work was funded by Wyeth and the National Heart Lung and Blood Institute of the National Institutes of Health, US Department of Health and Human Services. \\ For correspondence: $S$ Shumaker. \\ Department of Public Health Sciences, Wake Forest University Health Sciences, 2000 West First Street, Piedmont Plaza II, Winston-Salem, $N C$ 27104.E-mail:}

sshumake@wfubmc.edu

\section{COMMENTARY}

The Women's Health Initiative Memory Study (WHIMS) is a randomised double-blind trial designed to determine whether treatment with hormone therapy (conjugated equine estrogen $0.625 \mathrm{mg}$ and medroxyprogesterone acetate $2.5 \mathrm{mg}$ ) taken daily for four years would reduce the risk of all-cause dementia and of mild cognitive impairment in women over the age of 65 years. The neuroprotective effects of estrogen ${ }^{1}$ combined with evidence from observational trials of cognitive aging and the incidence of Alzheimer's disease in older women ${ }^{2}$ led to the expectation that hormone therapy would be associated with a lower incidence of Alzheimer's disease. In fact, the hazard ratio for probable dementia was twice as high in the hormone therapy group compared to the placebo group. Although there was no between-group difference in the rate of mild cognitive impairment, there was a small increased risk of cognitive decline over time in the estrogen plus progestin group compared to placebo.

Why did the WHIMS trial fail to confirm the putative beneficial effects of estrogen on mild cognitive impairment and incidence of Alzheimer's disease given positive findings from basic neuroscience and clinical studies? Several possibilities might be considered. First, most information on neuroprotection pertains to treatment with estrogen-alone. Indeed, there is evidence that medroxyprogesterone acetate attenuates ${ }^{4}$ or reverses $^{5}$ the beneficial effect of estrogen on psychological and cognitive function. We need to await the outcome of the unopposed arm of the WHIMS trial to determine whether medroxyprogesterone acetate was influential in the negative findings of the hormone therapy arm of this study.

Second, $80 \%$ of the WHIMS sample were hormone naïve, and started hormone therapy at a mean age of approximately 70 years. Both animal and human studies provide evidence that estrogen protects cognitive function when treatment is initiated soon after ovariectomy in rats ${ }^{6}$ or during the peri- or early postmenopause in women ${ }^{7-8}$ but does not provide cognitive protection when the onset of therapy is considerably delayed beyond the menopause. Because of the advanced age of its sample, data from WHIMS cannot address the possible protective effects of estrogen on cognition in younger, perimenopausal women, the segment of the population who are actually treated with hormone therapy in the real world.

What the WHIMS findings make clear is that 70 year old women should not be started on conjugated equine estrogen and medroxyprogesterone acetate for the purpose of reducing the risk of Alzheimer's disease or mild cognitive impairment. Since it has never been common practice to prescribe hormone therapy de novo for 70 year old women, these findings are unlikely to affect actual clinical practice. However, media reports, which have erroneously generalised the WHIMS findings to all women, will likely discourage perimenopausal women from initiating hormone therapy.

Professor Barbara B Sherwin, PhD and James McGill Department of Psychology E Department of Obstetrics and Gynecology McGill University, Montreal, Canada

McEwen BS. Estrogen action throughout the brain. Recent Prog Horm Res 2002;57:357-384.
2 Sherwin BB. Estrogen and cognitive functioning in women. Endocrine Rev. 2003;24:133-151.

Rapp SR, Espeland MA, Shumaker SA et al. Effect of estrogen plus progestin on global cognitive function in postmenopausal women. The Women's Health Initiative Memory study: a randomized controlled trial. JAMA 2003; 289: 2663-2672. Sherwin BB. The impact of different doses of estrogen and progestin on mood and sexual behaviour in postmenopausal women. $J$ Clin Endocrinol Metab. 1991;72:336-343.

Rice MM, Graves AB, McCurry SM et al. Postmenopausal estrogen and estrogen-progestin use and 2-year rate of cognitive change in a cohort of older Japanese American women; the Kane project. Arch Intern Med 2000;160:1641-1649.

Gibbs RB. Long-term treatment with estrogen and progesterone enhances acquisition of a spatial memory task by ovariectomized aged rats. Neurobiol Aging $200 \cdot 21: 107-116$

Matthews KA, Cauley J, Yaffe K, Zmuda J. Estrogen replacement therapy and cognitive decline in older community women. J Am Geriatr Soc 1999;47:518-523.

8 Zandi PP, Carlson MC, Plassman BL et al. Hormone replacement therapy and incidence of Alzheimer's disease in older women: the Cache County Study.J Am Med Assoc $2002,288.2123-2129$. 